\title{
La tesis imposible
}

\author{
Horacio Potel ${ }^{1}$
}

\begin{abstract}
Tras algunas observaciones amistosas sobre la conferencia que yo acababa de pronunciar, Jean Hyppolite añadía: «Aparte eso, verdaderamente no veo adónde va usted». Creo haberle contestado poco más o menos lo siguiente: «Si viese claramente, y por anticipado, adónde voy, creo realmente que no daría un paso más para llegar allí». Quizás pensé entonces que saber adónde se va puede indudablemente ayudar a orientarse en el pensamiento, pero no ha hecho jamás dar un paso, todo lo contrario. ¿Para qué ir adonde se sabe que se va y adonde se sabe uno destinado a llegar?
\end{abstract}

Jacques Derrida, El tiempo de una tesis

\section{Introducción}

En estas frases de Jacques Derrida, pronunciadas en su defensa de tesis, están expuestos los temas más importantes a la hora de mostrar la imposibilidad de la Tesis. La tesis es imposible, es imposible como acontecimiento, es decir como la llegada de lo otro, como invención del otro. Ya que para Derrida, una llegada programada, un acontecimiento programado, no es un acontecimiento. Es la repetición de lo mismo, la tesis programada, el programa de tesis es el filtro para impedir la llegada de lo nuevo. Pero la Tesis nunca llega.

\section{Trataremos de explicarlo mejor:}

Decir lo que la deconstrucción sea, es, claro, traicionarla, congelarla, asesinarla. Esto, desde ya, por supuesto, pasa con cualquier cosa: todo encasillamiento, encierra, encapsula, reduce, ya que supone una tarea de selección, de edición, en la que la totalidad e individualidad de "la cosa" -si es que algo así existe- necesariamente se pierde. Ahora bien, cuando la cosa a cercar, a limitar con un aparato conceptual, es la deconstrucción misma, la cuestión se vuelve paradójica: ya que la deconstrucción se empecina en mostrar que las cosas no tienen ni un significado definible ni una misión determinada. Que cada vez que se intenta definir una cosa, encerrarla en un casillero, la cosa misma se escapa y se escapa, porque lo que ocurre con las cosas no es algo que tenga que ver con el presente: el significado, la misión de las cosas, lo que ocurre con las cosas, siempre está por venir. La deconstrucción es una apasionada, amorosa, afirmación de lo por-venir. Esto quiere decir que lo que se afirma no es lo que es, lo que está, lo dado, lo presente, 1 Horacio Potel es Arquitecto y Licenciado en Filosofía por la Universidad de Buenos Aires. Se encuentra terminando su Tesis de Doctorado en Filosofía en la Universidad Nacional de Lanús. Es Profesor de Ética en la misma universidad. Ha publicado diversos textos, en la Argentina y en el exterior, donde trabaja algunos de los conceptos claves de Jacques Derrida, como herencia, diseminación, archivo, survie. 


\section{0/ Perspectivas Metodológicas /19/Vol. II /Año 2017}

eso que los enemigos de la esperanza suelen llamar "Lo Real". Lo que se afirma, por el contrario es lo por venir, y no lo por venir que algún día llegará, al fin de algún camino o como resultado de algún programa, de tesis por ejemplo. Lo por venir está siempre por llegar, lo que quiere decir que jamás se consumará en presente alguno. Lo por venir es lo que no se ve en ningún horizonte, lo monstruoso, lo imprevisible más allá del horizonte de lo mismo. La deconstrucción es una afirmación infinita, afirmación sin descanso ni meta, ni tesis final, de lo que está en construcción deconstruyéndose. La afirmación de lo otro que viene, la invención de lo otro, la promesa siempre renovada. Promesa originaria, pues si estoy constituido por lo otro, porque no soy el primero porque no hay primero, es la interpelación de lo otro lo primero, antes que cualquier pregunta, antes que cualquier teoría, antes que cualquier filosofía está lo otro, al que empiezo, antes de empezar y de ser, respondiendo: sí, sí.

Pero esto, el encasillamiento, es de alguna manera lo que nos pide el aparato de control académico, cuando nos exige tesis y artículos, es decir, tener por lo menos UNA posición firme que delimite y encierre en su cierre a la tesis, o a una tesis sobre Derrida. El cual, por cierto, también en ocasión de su defensa de tesis, decía: "La idea misma de presentación tética, de lógica posicional u oposicional, la idea de posición, [...] era una de las piezas esenciales del sistema sometido a un cuestionamiento deconstructor". ${ }^{2}$ Para Derrida "resumir o presentar conclusiones téticas" es imposible. Como ya dice en uno de sus trabajos juveniles: "Una de las tesis -hay más de una- inscritas en la diseminación es justamente la imposibilidad de reducir un texto en cuanto tal a sus efectos de sentido, de contenido, de tesis o de tema". ${ }^{3}$ Pareciera, entonces que una tesis, una posición sobre la deconstrucción, es una tarea condenada al fracaso, una tarea imposible. Pero he aquí que la deconstrucción es una incesante espera de lo imposible es decir de aquello cuya posibilidad está sostenida por su imposibilidad. Pues bien, como la lealtad pura es imposible, vamos a empezar con la traición impura, en nombre de una fidelidad infiel, para poder explicar tantas imposibilidades. Entonces lo mejor será que comencemos. Lo que no borra el dolor, ni el sentimiento de la traición; traición porque es imposible terminar, porque el contexto está siempre abierto, porque siempre está abierto el porvenir, y entonces jamás se podrá pronunciar la última palabra. Pero ese es, justamente, desde siempre el precio a pagar si se dice algo, la imposibilidad de decirlo todo, la certeza de estar en la incerteza, la impureza del perjurio, de la infidelidad, de la traición. Y a la vez, otra vez, a la vez, la traición, la infidelidad y el perjurio, es lo único que pude conservar el secreto, y el secreto es justamente lo que permite que nunca termine una tesis, un texto, una vida. Que nunca se cierre en una totalidad, que nunca baje a su tumba. Nada ni nadie termina nada ni nadie. Ni la muerte termina. Y entonces de nuevo lo único que se puede hacer es comenzar. Comenzar no para concluir nada, comenzar para inscribir la inconclusión, comenzar para sumar un trazo, un resto , algo que comienza ya como ruina y ceniza, y que justamente solo en tanto resto y ceniza abre el por-venir.

Comparemos la compulsión de tesis, esa obsesión por tener una posición, una estabilidad, un pensamiento original, con por ejemplo la avaricia.

2 Derrida, J., El tiempo de una tesis. Deconstrucción e implicaciones conceptuales, Proyecto A Ediciones, Barcelona, 1997, pp. 11-22. Texto presentado en la sesión de defensa de la tesis, basada en las obras publicadas de Derrida, que tuvo lugar en la Universidad de la Sorbonne, el 2 de junio de 1980, ante un tribunal formado por los profesores Aubenque, De Gandillac, Desanti, Joly, Lascault y Lévinas.

3 Derrida, J. «Hors libre», en La Diseminación, trad., J. Arancibia (mod.), Madrid, Fundamentos, 1997, p. 13. 
Afán de poseer para atesorar. Tal es una de las definiciones más usuales que suelen dar los diccionarios para hablar de la avaricia. Parecería entonces que el avaro es el incapaz de dar. Encerrado en sí mismo el avaro construiría una pared, un cerco, un búnker, una cripta en donde encerrar sus tesoros, en donde encerrar su sí mismo avaro, parecería... Pero, ahora bien, estos tesoros que atesora el avaro: ¿De dónde vienen? ¿De dónde sale eso que guarda con tanto afán?

Si el avaro no es el Dios de la ontoteología -hipótesis que hoy no abordaremos- es decir, si el avaro no es causa sui, inegendrado, completo, lleno, perfecto y puro, entonces esos tesoros deben haber sido obtenidos de otro lado que no sea la cripta, el búnker, el encierro del avaro; deben de proceder por tanto de allende las fronteras de su mismidad.

El avaro necesita, pues, de lo que está afuera de su encierro, más allá de sus fronteras, para poder ser así avaro, necesita de eso otro que no tiene, y lo necesita desde el primer momento. No es que sólo lo necesita para acrecentar su tesoro, necesita de lo otro para poder ser aún antes de ser avaro, necesita de lo que no tiene para tener, atesorar y no dar, o al menos intentar no dar. Necesita de lo otro, de lo que no es él para ser él, su avaricia necesita apropiarse de eso que no le pertenece, debe afanarse para que entre algo de lo otro en su más profunda mismidad para poder aspirar a ser él mismo. Es decir que el avaro está habitado desde siempre por lo que no le pertenece, por lo que él no es, por otro que es a la vez su riqueza y la segura fuente de su ruina.

La avaricia perfecta, la avaricia pura no puede entonces nunca constituirse, porque desde el primer momento está habitada por lo que no es, por un exceso que es justamente su contario pero al que está ligada de manera irreductible. Con lo cual ya se sentenció el fracaso de la avaricia. El avaro necesita tomar, apropiarse del otro, pero esa apropiación nunca puede ser completa, es necesario que la apropiación falle, que reste siempre algo de alteridad, para que el mecanismo de la avaricia pueda seguir funcionando.

La avaricia pura, si la hubiera, sería el cierre total en la totalidad del todo. El avaro se convertiría en el UNO, en el TODO, privado de toda alteridad, de toda diferencia, inmóvil a falta de espacio, incrustado en el instante sin tiempo de una eternidad eternamente presente, eternamente igual, eternamente la misma. El Todo es lo que no tiene exceso, el Todo no da. El avaro, ahogado, oprimido en su sí mismo, ya no sería ni siquiera un avaro; no tendría nombre, porque sería todos los nombres o la falta de todos ellos, ya que no hay nada que nombrar en el Todo. Avaro sin afán posible, ya que no podría apropiarse de ninguna alteridad, sin nada que atesorar porque no hay nada ni nadie que le pueda quitar ni dar nada. Porque la avaricia perfecta, o la totalización del Todo, sería a la vez que la muerte del avaro, la muerte del afuera, la muerte del otro -por el cual el avaro era-; el otro dejaría de ser otro para ser el mismo, o lo que es lo mismo, el mismo dejaría de ser el mismo para pasar a ser el otro, con lo cual ya no habría ni mismo ni otro: sólo Todo. Ni avaro ni tesoro: Todo.

Si pensamos el origen a la manera tradicional como origen simple, pues entonces no hay origen, ya que del uno como hemos visto, no sale nada. El Uno-Todo no da nada. Si 


\section{2/ Perspectivas Metodológicas /19/Vol. II / Año 2017}

el origen está contaminado por la alteridad entonces, como dice Derrida en su artículo sobre Freud: "Es el no-origen lo que es originario". Si en el origen están el mismo y el otro coimplicados, no puede haber primero, porque el primero necesita del segundo, para poder ser primero, pero entonces el segundo es la condición de posibilidad del primero, el retardo del segundo es lo que le permite al primero ser primero, pero entonces no lo es, como tampoco, claro está, y por los mismos motivos, no puede ser primero el segundo. Como decíamos hace un segundo si la plenitud, si la identidad no está dada, es necesario que algo supla la falta y este suplemento es originario. La repetición es originaria.

El otro entonces me antecede, me sucede, me constituye, me destituye, me acosa, me inventa. Y esta relación con lo otro es para Derrida, como sabemos, la Justicia. Y es la justicia la que nos impone el deber de abrir las fronteras, de romper con la avaricia en una hospitalidad incondicional ante la llegada del otro en cuanto otro. Como otro absoluto. Este deber (que lo otro siga siendo otro) es el que determina las condiciones para que sea posible lo que Derrida llama Acontecimiento, es decir la llegada del por-venir. Entonces para que las cosas ocurran, para que sigan dándose eventos, es preciso que lo otro reste, resista como otro. La paradoja aquí es que la llegada del acontecimiento, la hospitalidad incondicional, el don -de ser posibles- de presentarse algún día a la luz de la presencia terminarían con todo otro, como también vimos hace un instante. La llegada del Acontecimiento, si fuera posible, sería la detención de todo, la catástrofe última, pero tal cosa es imposible. No hay principio y no hay fin. El UNO es la cancelación de la sobrevida, que necesita siempre para darse del juego, del resto, del fracaso en la apropiación, del fracaso de la avaricia de una cierta indemnidad. El UNO es la Tesis, el cierre, el sentido, lo que por suerte nunca puede hacerse presente asegurando así el por-venir. Una cierta indemnidad de la alteridad absoluta, lo que Derrida llama a veces: lo indeconstruible, es decir el resto siempre resta. Es lo que siempre escapa en un proceso de apropiación, académico, por ejemplo.

La lectura de Husserl llevo a Derrida a sostener que no se puede acceder a las cosas mismas, es decir a una conciencia pura y a un objeto no constituido, mediado y diferido. No es extrańo que desde este planteo se piense el acceso a una alteridad radical como imposible. Y es que para Derrida, no puede haber un afuera puro limitando con un adentro puro: el afuera esta dentro del adentro, lo otro habita en el mismo, el mismo está en lo otro y el adentro está contaminado por el afuera, desde siempre. Exceder el discurso de la filosofía, que siempre se ha ceñido aasegurar el dominio del límite, no puede significar dar un paso fuera de la clausura. Pensar «Una clausura de la representación cuya forma no podía ser ya lineal, indivisible, circular, enciclopédica o totalizante» ${ }^{4}$ es pensar la supervivencia de los envíos. Porque la presencia es imposible, la re-presentación está clausurada, esto quiere decir que "no hay fuera de texto", ${ }^{5}$ si la survie es "todo aquello con lo que está entretejido de arriba abajo, el tejido de la existencia", no hay fuera de la sobrevida, lo que no quiere decir que la sobrevida o mejor las sobrevidas sean inmortales, no sólo por la finitud de la huella, como ahora veremos, si no sobre todo porque la sur-

4 «Envío», op.cit.

5 Derrida, J.: De la gramatología, trad. de O. del Barco y C. Ceretti, México D.F., Siglo XXI, 1998, p. 202. Cfr. p. 203: «Dentro de lo que se llama la vida real [...] nunca ha habido otra cosa que escritura; nunca ha habido otra cosa que suplementos, significaciones substitutivas que no han podido surgir sino dentro de una cadena de reenvíos diferenciales». 

vie jamás termina de constituirse: un texto, cualquier texto, oculta siempre su sentido, porque éste está siempre por venir, lo que también quiere decir que nunca puede llegar a un destino final, salvo que la huella desaparezca y que su tejido se pierda sin que nadie sepa nunca que se perdió, porque nunca se constituyó, nunca se encontró, eso perdido.

Si no hay presencia plena, no puede haber un "presente" el tiempo mismo no puede hacerse presente. No hay algo así como un "presente viviente" y no sólo por la imposibilidad de la presencia de constituirse como tal, sino porque como acabamos de ver: que la presencia nunca pueda llegar a estar presente hace que ésta deba desde siempre ya repetirse, re-presentarse en un suplemento, en un artefacto técnico. La vida, entonces, es habitada por la muerte, o la muerte es habitada por la vida desde siempre ya. Esta coimplicación originaria es la survie. Con lo cual no solo no podemos decir que seamos "vivos" o "muertos": somos fantasmas, sobrevivientes asediados por la vida y por la muerte; sino que también, y también esto es otra forma de decir lo mismo, no podemos decir que somos humanos o vivientes en general opuestos a máquinas; ${ }^{6}$ el suplemento de origen implica que la máquina está también desde siempre ya en la vida, con lo cual esta no puede pretender nunca estar desnuda o pura y ajena a la repetición, al retraso, a la muerte. Somos cyborg. Que la "vida" esté desde siempre coimplicada con la "muerte", significa también que la "vida" está desde siempre coimplicada con lo no maquinal, con el acontecimiento.

Que la survie no pueda empezar ni terminar no quiere decir que la survie sea infinita, la survie es indefinida. Toda huella es finita, toda huella debe poder perderse, desaparecer, no hay presencia sin huella ni huella sin muerte. "Una huella imborrable no es una huella, es una presencia plena, una sustancia inmóvil e incorruptible, un hijo de Dios, un signo de la parousíay no una semilla, es decir, un germen mortal". ${ }^{7}$

Lo que es imposible es la Muerte Absoluta, que de darse sería a la vez la Vida Absoluta. La conclusión de la Tesis, la presentificación del Mesías cerraría, envolvería en el círculo de lo mismo al tiempo y a la historia, sería el fin de la promesa y por tanto el fin de la esperanza, el fin del deseo, el fin de la pasión, el fin del futuro, el fin de la vida, el fin de toda sobrevida. La incondicional llegada del otro, de ser posible, sería la muerte del otro convertido por este acto en el Mismo, privado de toda diferencia; La Muerte, que es también lo imposible. La promesa mesiánica es una promesa que se rompería si se cumpliera, la posibilidad del Mesías es su imposibilidad. Para mantener la esperanza es necesario romper con toda esperanza determinada. Y seguir jugándose por lo imposible.

El resto es la condición de posibilidad del acontecimiento y la filosofía de Derrida es una filosofía del acontecimiento, entendiendo por tal: la donación de lo otro, que en tanto otro no puede ser lo mismo, y en cuanto don no puede ser lo dado, lo que implica la improgramabilidad del acontecimiento.

6 Cfr. De la gramatología, op. cit., p. 15: "Aún suponiendo que la teoría de la cibernética pueda desprenderse de todos los conceptos metafísicos -hasta del concepto de alma, de vida, elección, memoria- que anteriormente han servido para oponer la maquina al hombre, tendrá que conservar [...] la noción de escritura, de huella, de grama o de grafema. Incluso antes de ser determinado como humano [...] o como a-humano, el grama -o el grafema- dará así el nombre al elemento. Elemento sin simplicidad". El subrayado es nuestro.

7 «Freud y la escena de la escritura» en La escritura y la diferencia, op. cit., p. 315. 
¿Puede un sujeto responder de sí? Para hacerlo debería saber todo sobre sí, debería tener un "yo" que acompañe todas "sus" representaciones, debería ser capaz de totalizarse bajo un solo y único nombre, pero esta identidad consigo mismo está permanencia calculable, este encierro, si fueran posibles, haría imposible todo acontecimiento, tornando la decisión algo accidental que no cambia nada, que no abre nada. Si el acontecimiento es lo que viene sin ser previsto, predeterminado, pronosticado, el acontecimiento de la decisión no me pertenece, es "lo otro en mí, que decide y desgarra" ${ }^{8}$ Si la decisión está en mi(y estamos viendo cuántas dificultades conlleva este mí) si se encuentra bajo misaber y mi poder, si la decisión es para mí posible, si es una decisión que yo puedo tomar, siendo entonces el paso al acto de mis posibles, pues entonces no decido nada. Al pasar quiero mencionar que nos encontramos nuevamente con lo imposible como condición de posibilidad, la posibilidad de la decisión está sostenida en su imposibilidad. La decisión debe ser entonces una decisión pasiva, la decisión del otro en mí, decisión inconsciente que sin embargo no me libra de ninguna responsabilidad, ni de ninguna libertad. Ya que hay por venir, el contexto está siempre abierto porque acontece, si el contexto siempre está abierto entonces nunca puede ser del todo determinable. Que no se pueda saber nunca todo sobre algo, garantiza que en cada caso, me encuentre ante la necesidad de decidir lo indecidible, que no esté obligado por un cálculo o un programa sino entregado a la locura del instante, porque como dice Kierkegaard "El instante de la decisión es una locura", locura que nos arroja al desgarramiento de tener que elegir sin ninguna seguridad. La decisión como imposible debe ser indecidible. "Indecidible es la experiencia de lo que siendo extranjero, heterogéneo con respecto al orden de lo calculable y de la regla, debe sin embargo -es de un deber de lo que hay que hablar- entregarse a la decisión imposible". ${ }^{9}$ Indecidible no tiene nada que ver con indecisión, ni con parálisis.Lo indecidible es la condición de la decisión, del acontecimiento.

Lo contrario de la indecidibilidad no es la decisión sino lo calculable, lo programable, lo formalizable, lo computable. En este sentido decidir algo decidido no es decidir nada, es nuevamente asegurar el círculo de lo mismo. Sin el riego de esta indeterminación no habría lugar para el deseo y por tanto tampoco para el porvenir. Es entonces, para Derrida, el momento de ser fiel-infiel a la herencia de Kant, siguiendo hasta el extremo su propia lógica: para Kant una acción moral no debe solamente ser "conforme al deber", sino que debe ser llevada a cabo "por deber", "por puro deber". Muy bien, dice Derrida, pero la cosa no termina aquí; si yo actúo por puro deber, es decir, porque debo, no puedo afirmar que mi acción sea moral: en primer lugar dicha acción está determinada por un saber: yo sé lo que se debe hacer, cuento con una regla o un programa, una receta que me dice qué es lo que debo hacer. Y por otro lado si actúo porque debo, si el motivo de mi acción es una deuda, mi acción consiste en cancelar dicha deuda, en cerrar el círculo económico de un intercambio comercial, quedándome entonces encerrado en una totalización, en una reapropiación, que el acontecimiento de la decisión debería desbordar. La moralidad pura debe exceder todos los cálculos conscientes o inconscientes, todos los propósitos, todos los proyectos de restitución o de reapropiación. La decisión debe permanecer indecidible, nunca puede

8 Derrida, J.: Políticas de la amistad, trad. De Patricio Peñalver y Paco Vidarte, Madrid, Trotta, 1998, p. 87.

9 Derrida, J.: «Del derecho a la justicia», en Fuerza de ley. El «fundamento místico de la autoridad», Madrid, Tecnos, 1997, p. 55. 
decirse de ella que está presente y que es justa. En efecto: o fue tomada sin ninguna regla y nada permite entonces decir que es justa, o fue tomada de acuerdo con una regla que nada garantiza o bien en el caso de que la regla garantizara la justicia de la decisión ya no se trataría de una decisión sino de un cálculo. Con lo cual el fantasma de lo indecidible acosa a toda decisión deconstruyendo toda seguridad de presencia, toda buena conciencia, la existencia misma de la decisión en cuanto tal. El deber de lo indecidible pone en evidencia lo engańoso de basar una ética en el sujeto: "Sin duda la subjetividad de un sujeto, ya, no decide nunca sobre nada: su identidad consigo y su permanencia calculable hacen de toda decisión un accidente que deja al sujeto indiferente. Una teoría del sujeto es incapaz de dar cuenta de la menor decisión. [...] lo otro en mí que decide y desgarra. La decisión pasiva, condición del acontecimiento, es siempre en mí, estructuralmente, otra decisión, una decisión desgarradora como decisión del otro. Del otro absoluto en mí, del otro como lo absoluto que decide de mí en mî". ${ }^{10}$

Habíamos pensado terminar acá este escrito, ya que es un texto que habla sobre la imposibilidad de la tesis, sobre como no se puede escribir nunca todo sobre nada. Un texto que habla sobre la imposibilidad del fin y la deriva indefinida del sentido no debería encontrar de pronto un sentido al final del texto. Pero para seguir con las indicaciones académicas -indicaciones que se presentan como sí de exigencias "neutrales" se tratara, llenas de "buena fe" y avaladas por la palabra "método", trataremos acá de dar cierre, fin, sentido, resumen, es decir, todas esas cosas que está tradición no cuestionada de la Tesis, da por sentado que se encuentran tras un largo camino, al final del mismo, en una lógica teleológica que nos cansamos de recusar pero en la cual seguimos escribiendo como si fuera lo mismo como si no importara, como si la escritura fuera sólo un vehículo tosco, una herramienta o un ornamento que no hace a la survie del texto. En definitiva escribimos tesis contra el logocentrismo pero escribimos metafísicamente, bajo la metafísica del logocentrismo: que piensa que un texto no vale por sí mismo, que la "IDEA" está siempre más allá del texto, más allá de su concreta escritura. Entonces tendremos que hacer como si todo lo escrito no hubiera servido de nada, como si todo lo que se dijo sobre la imposibilidad del cierre y el fin, pudiera ser envuelto por una forma de escribir teleológica. El logocentrismo metafísico es el privilegio dado a la palabra viva, sobre la escritura técnica. Una ideología que para ocultar el valor de las normas, de los rituales burocráticos, de los formularios y las reglas de escritura, plantea el pensamiento como libre de toda estructura técnica, de toda pro-tesis. Así el plan de tesis, o el abstract, por ejemplo, deben decir lo que dirá la tesis antes de ser escrita y como la tesis comprende o debe comprender en sí misma la totalidad del sentido: el plan que planea la tesis es la mejor tesis; con lo que no se entiende la necesidad de escribirla. Según esta narrativa sabemos antes de escribir lo que vamos a decir, lo sometemos a la escritura concebida como un proceso de despliegue de esa totalidad que es la IDEA que vamos a manifestar para por fin terminar con el mejor resumen posible de esa IDEA que siempre, según este relato va a estar mejor en nuestro pensamiento que sometida a las reglas del aparato técnico de la escritura que justamente por estar hundido en lo técnico no puede jamás expresar en "verdad", en carne y hueso espirituales, el éter de las ideas.

10 Derrida, J., Políticas de la amistad, trad. De Patricio Peñalver y Paco Vidarte, Madrid, Trotta, 1998, pp. 86. 
El programa, el método es siempre protección contra el acontecimiento. Un programa perfecto programa perfectamente todo. Lo por hacer está ya hecho antes de hacerse, con lo cual no está por hacerse sino que se ha hecho. Los métodos académicos son programas burocráticos para reproducir las condiciones de la vida académica, sus jerarquías, sus pequeños privilegios.

Con esta teleología del sentido último hace pareja el mito del "autor", nosotros no comulgamos con la fe de esta creencia, no somos Dios, no hay nada de original en lo que escribimos, ya que no hay origen. La escritura se independiza del escritor desde el primer momento, escrita, ya no necesita de él, vive sin él. Se independiza desde siempre de su supuesto autor para devenir máquina productora, diseminante del sentido, separada de la conciencia y por tanto de las intenciones y de la plenitud del querer-decir de éste, y de cualquier otro que quiera erigirse en el dueño, o el restaurador de un supuesto sentido originario.

La unidad del texto, esta unidad que venimos cuestionando, asegurada supuestamente por la firma de un autor, necesita de la contra-firma del otro. Pero como todo otro, todo destinatario determinado, todo acto de lectura, se encuentra marcado por la misma muerte, la lectura no tiene fin, está siempre por venir, y por venir del otro. El cierre del sentido se vuelve así imposible. La escritura no pertenece al reino de la presencia, por el contrario es la condición de posibilidad de ésta. Al no pertenecer a la presencia no corresponde preguntarse por la esencia de la escritura, sino por la forma en que la escritura se inscribe, se marca, se traza y lo hace repitiendo, repitiéndose, iterándose. Diseminándose en una multiplicidad irreductible, la ausencia rompe el límite del texto, con lo cual queda impedido su totalización y su cierre, nunca acaba el querer-decir, la firma siempre está abierta a una nueva contrafirma. La herencia no termina. ¿'Terminamos? con Derrida:

"La fuerza reproductiva de la autoridad se conforma más fácilmente a declaraciones o tesis sedicentes revolucionarias en su contenido codificado con tal que se respeten los ritos de legitimación, la retórica y la simbólica institucional que desactiva y neutraliza todo lo que viene de otra parte. Lo irrecibible es aquello que, por debajo de las posiciones o las tesis viene a trastornar ese contrato profundo, el orden de esas normas, y que lo hace ya en la forma del trabajo, de la enseñanza o de la escritura”.11

\section{Bibliografía}

- Derrida, J.: «Del derecho a la justicia», en Fuerza de ley. El «fundamento místico de la autoridad», Madrid, Tecnos, 1997.

- Derrida, Políticas de la amistad, trad. De Patricio Peñalver y Paco Vidarte, Madrid, Trotta, 1998.

- Derrida, J.: De la gramatología, trad. de O. del Barco y C. Ceretti, México D.F., Siglo XXI, 1998.

- Derrida, J., El tiempo de una tesis. Deconstrucción e implicaciones conceptuales, Proyecto A Ediciones, Barcelona, 1997.

- Derrida, J. «Hors libre», en La Diseminación, trad., J. Arancibia (mod.), Madrid, Fundamentos, 1997

11 Derrida, J.: "El tiempo de una tesis: puntuaciones", op.cit, pp. 18. 